\title{
Judicialización de las internaciones psiquiátricas involuntarias: nuevos paradigmas y responsabilidad judicial.
}

Judicialization of involuntary psychiatric hospitalizations: new paradigms and judiciary responsibility.

\section{Claudia Elizabeth Zalazar}

Abogada. Magistrada en el Poder Judicial. Córdoba, Argentina.

\section{Sofía Puccio}

Abogada. Programa de Direito Sanitário da Fundação Oswaldo Cruz. Brasília, Brasil.

Resumen: La nueva ley de salud mental en la República Argentina que regula la protección de los derechos de las personas con padecimiento mental, ha sentado como un paradigma fundamental la desjudicialización del sistema, entendido en un doble sentido: como una limitación de las facultades judiciales a la hora de evaluar la posibilidad de dicha medida restrictiva, la que sólo debe ser aplicada como último recurso terapéutico tras una valoración interdisciplinaria y en segundo lugar como control efectivo que la duración de dicha internación sea por el menor tiempo posible; siempre a la luz de la protección de los derechos humanos de dicho paciente. Si bien la normativa nacional se ajusta a los principios de las normas internacionales la misma no ha sido acompañada por políticas de salud mental que garanticen en la práctica su cumplimiento, ocasionando distorsiones en su aplicación, que deben ser corregidas; con la consiguiente responsabilidad del Estado de manera general y del Poder Judicial en forma particular ya que es en definitiva el que tiene que bregar por el cumplimiento de dicha ley.

Palabras-clave: Judicialización; salud mental; políticas de salud; Poder Judicial, responsabilidad.

Resumo: A nova lei de saúde mental na República Argentina, que regula a proteção dos direitos das pessoas com doenças mentais, firmou-se como um paradigma fundamental para a desjudicialização do sistema, sendo analisada sob duas vertentes: em primeiro lugar, pelas limitações das atribuições judiciais à hora de avaliar a possibilidade da medida restritiva, que só deve ser aplicada como último recurso terapêutico, após uma avaliação interdisciplinar. Por outro lado, preocupa-se com o controle efetivo da duração do internamento, para que este seja pelo menor tempo possível, sempre à luz da proteção dos direitos humanos do paciente. Apesar de a legislação nacional está em conformidade com os princípios das normas internacionais, este processo não foi acompanhado pelas políticas de saúde mental, responsáveis por garantir, na prática, o cumprimento deste direito. São causadas distorções na aplicação da lei, que devem ser corrigidas com a responsabilidade geral do Estado e, em particular, do Poder Judiciário, que tem que se esforçar para garantir o cumprimento destas leis.

Palavras-chave: Judicialização; saúde mental; Poder Judiciário, responsabilidade. 
Abstract: The new health's law in Argentina Republic that regulates the protection of the insane people's rights has changed a fundamental paradigm of the no judicialization of the system, which is understands in two ways: like a limitation of judiciary powers at the moment of evaluations the possibilities of that restrictive decision, that only has to be take like the last therapeutic resource beyond the interdisciplinary evaluation and in the other hand like a effective control about the time of the hospitalization, which has to be minimum, always under the light of human rights. Even though the national law adjusts at the values of international laws, the regulation hasn't been following by mental health's politics which guarantee the practice, generating distortions at the moment of the application, that will be corrected, with the State's responsibility in general way and of the Judiciary Power particularly, because it is the part of the State that has to do that the people observe the law.

Keywords: Judicialization; mental health; health policies; Judiciary Power, liability.

\section{Presentación del tema}

De manera preliminar debemos subrayar que, conforme a los "Principios para la Protección de los Enfermos Mentales y el Mejoramiento de la Atención de la Salud Mental" (en adelante Principios de Salud Mental), adoptados por la Asamblea General de las Naciones Unidas en su Resolución № 46/119, del 17 de diciembre de 1991, una persona puede ser admitida como paciente involuntario cuando queda acreditado que padece una enfermedad mental grave, que su capacidad de juicio está afectada y el hecho de que no se la admita o retenga en una institución psiquiátrica pueda acarrearle un gran deterioro de su condición (Principio 16, inciso 1).

Bajo la vigencia del antiguo art. 482 del Código Civil Argentino y de la Ley $\mathrm{N}^{\circ} 22.914$ y de las normas reglamentarias que fueron dictándose a los fines de implementar el sistema de las llamadas "internaciones judiciales involuntarias", el número de pacientes institucionalizados por orden judicial creció sensiblemente; sea porque la orden de internación provino de una denuncia formulada ante un tribunal o bien por el hecho de que las iniciadas como voluntarias, policiales o de urgencia, necesariamente se convertían luego en judiciales, ya sea por falta de respuesta del sistema de salud o por ignorancia de las autoridades administrativas. 
En el contexto expuesto, se suscitó un arduo debate acerca de la compatibilidad entre la desjudicialización de las internaciones y la protección del paciente institucionalizado, finalidad ésta última que ha sido consagrada por la legislación internacional vigente en nuestro país ${ }^{1}$, habiendo propuesto algunos sectores la creación de órganos de revisión específicos compuestos por profesionales del Derecho, de la Salud y miembros de la comunidad que no revistan dichas calidades (Avalos, 2008) y que culminaron con la sanción de la nueva Ley $n^{\circ}$ 26557, que legisla sobre "derecho a la protección de la salud mental" la que analizaremos posteriormente.

Con relación al antiguo sistema imperante en Argentina, el hecho de que la totalidad de requerimientos relacionados con internaciones psiquiátricas, así como el movimiento en torno a quien padece dicho trastorno - externación, permisos de salidas, altas provisorias etc. - se canalizara a través del órgano judicial, resultaba un verdadero inconveniente para el Servicio de Justicia y mucho mayor para el justiciable.

Dicho sistema de internaciones psiquiátricas involuntarias había logrado cambiar el eje de la responsabilidad, ya que cuestiones que debían ser decididas por los expertos en salud - y en especial en salud mental (valorando las distintas terapias previas a una internación) - terminaban siendo resortes del poder judicial, con pericias psiquiátricas forenses urgentes y distorsionadas, ${ }^{2}$ no cumpliéndose adecuadamente con ninguna de las normas sobre cuidado y seguridad del paciente mental.

El problema que debía ser, en principio, de salud pasó a ser judicial, con todas las implicancias que ello traía aparejado: pericias hechas por los médicos forenses que formaban parte del Poder Judicial en la alcaldía de Tribunales, traslados de los pacientes en autos policiales, negativa de los hospitales a su recepción, internaciones voluntarias que se judicializaban ante el rechazo de las instituciones públicas por falta de camas etc.

\footnotetext{
${ }^{1}$ Así, por ejemplo, de acuerdo a los Principios para la Protección de los Enfermos Mentales y el Mejoramiento de la Atención de la Salud Mental, adoptados por la Asamblea General de las Naciones Unidas en su Resolución № 46/119 del 17/12/1991, una persona puede ser admitida como paciente involuntario cuando queda acreditado que padece una enfermedad mental grave, que su capacidad de juicio está afectada y que el hecho de que no se la admita o retenga en una institución psiquiátrica pueda acarrearle un gran deterioro de su condición (Principio 16 inc. 1).

2 El perito contaba con escasa información sobre el paciente, en lo que respecta a sus antecedentes siquiátricos.
} 
En definitiva, la internación judicial involuntaria, que debía ser de excepción, se había convertido en la forma ordinaria de contener a las denuncias de personas con trastornos mentales en un supuesto "estado de peligrosidad”, confundiéndose muchas veces el diagnóstico de peligrosidad paciente que puede ocasionar daño severo para sí o para terceros y que requiere inmediata internación (homicidio, suicido, agresión física, delitos sexuales etc.) - con un pronóstico de peligrosidad - paciente que necesita un tratamiento psiquiátrico adecuado de su enfermedad y con la contención adecuada, pudiendo resultar eventualmente peligroso para sí o para terceros. Este último no es pasible de una internación inmediata, menos aún involuntaria, pudiendo recurrirse a las otras alternativas que establece el sistema de salud: internaciones domiciliarias, tratamientos ambulatorios, abordaje de equipos de salud comunitaria, programa de salud familiar etc.

A ello debíamos agregarle un tedioso y largo trámite para lograr la externación del paciente, en el que también se había invertido la responsabilidad al juez - cuando el que debe evaluar las condiciones de viabilidad de la misma es el mismo equipo de salud tratante - toda vez que tanto el alta sanatorial como los permisos provisorios, necesitaban de la orden judicial respectiva.

Esta realidad impuso reflexionar acerca de la admisibilidad y conveniencia del empleo de técnicas alternativas dentro de la tendencia global hacia la desjudicialización de los conflictos, por un lado, y la optimización de recursos en el área de la salud pública, por el otro, los cuales debían acompañar ese proyecto ya que, de lo contrario, la reforma se vería significativamente afectada (funcionalidad del sistema emergencista, provisión de ambulancias, capacitación de los médicos etc.).

Ello es así ya que en la mayoría de los casos, la judicialización es la contracara de un déficit en el sistema sanitario - no solo en materia de salud mental sino en el sistema de salud en general - que ha dado, en los últimos tiempos, a una proliferación de juicios de amparo contra obras sociales o el Estado para conseguir la aplicación del ciudadano a su derecho a la salud.

Así, a fin de tutelar la dignidad de la persona y facilitar la actuación coordinada y eficiente del Poder Judicial con los organismos del Estado, y con el claro objetivo de que la actuación judicial pueda centrarse en realizar su 
principal rol de custodio de los derechos fundamentales de los ciudadanos con patologías psiquiátricas, se propusieron modificaciones a los procedimientos para las internaciones por patologías psiquiátricas.

\section{Los nuevos paradigmas en Salud Mental}

La nueva Ley de Salud Mental (recientemente reglamentada mediante Decreto no 603, del 13 de mayo de 2013) ha sido concebida como un instrumento necesario para proteger los derechos de aquellos sujetos que experimentan algún padecimiento relacionado con su salud mental.

Estos últimos son sujetos especialmente vulnerables, quienes suelen enfrentarse a situaciones de estigmatización, discriminación y marginación, incrementándose así la probabilidad de que se violen sus derechos. De allí, que esta ley se enmarque en la protección de los Derechos Humanos, entendidos como una dimensión clave en el diseño, desarrollo, seguimiento y evaluación de los programas y políticas de Salud Mental. Estos incluyen, entre otros, los derechos a la igualdad; a la no discriminación; a la dignidad; al respeto a la privacidad y a la autonomía individual, a la información y a la participación (Ley 26.657, art. 7).

Es dable recalcar los fundamentos legislativos de dicha ley, los que expresan: En nuestros días, la problemática de la salud-enfermedad atención mental se visibiliza como un problema relevante para la salud pública y requiere ser abordado tanto en su especificidad como en forma integral como parte indisoluble del Derecho a la Salud y los Derechos Humanos en general de todas las personas.

Entre otras acciones, se propone la transformación institucional del sistema de salud mental, erigidos en dos grandes pilares: por un lado, la desmanicomialización, desinstitucionalización y desjudicialización de los pacientes que padecen trastornos mentales; $y$, por otro lado, el abordaje de los problemas de salud mental en todos los niveles asistenciales, como parte integrante de los servicios generales de salud y desde una óptica interdisciplinaria (Ley 26.657, art. 8, y nueva versión de los arts. 152 bis y 482 del Código Civil); haciendo especial hincapié en los derechos de los pacientes 
de la salud mental y en especial la necesidad de su consentimiento informado cuando fuera posible.

\section{a. Desmanicomialización y desinstitucionalización}

Para lograr estos objetivos, se establece la paulatina transformación de las instituciones y servicios de salud mental existentes en la actualidad como "monovalentes"; la creación de nuevas instituciones como hogares de medio camino, hospitales de día etc., y la admisión en los hospitales generales de personas con padecimiento mental por períodos breves. Asimismo, la prohibición de la creación de manicomios o instituciones monovalentes de internación prolongada, públicos o privados.

\section{b. Desjudicialización}

Se establece en la normativa nacional la limitación de la intervención judicial y la correlativa atribución de funciones al equipo interdisciplinario, lo que significa devolver al sistema de salud la responsabilidad en la atención del paciente con padecimiento mental. Según el art. 23 de la ley nacional, el alta, la externación y el permiso de salida es una facultad del equipo de salud que no requiere autorización judicial, medidas que sólo deben comunicarse al juez - salvo que se trata de un paciente que haya ingresado al sistema penal a través de una medida provisional o de seguridad en los términos del art. 34 del Código Penal, en cuyo caso se deberá contar siempre con la orden judicial correspondiente.

Asimismo, se crea el órgano de revisión a quien se le confiere facultades especiales para merituar las decisiones judiciales y en su caso pedir la sanción de los jueces en las situaciones que hubiera irregularidades como denunciarlos ante el Consejo de la Magistratura o el organismo que controle la conducta de los mismos en las jurisdicciones provinciales (vgr. en Córdoba el Jury de Enjuiciamiento).

Con relación a las internaciones psiquiátricas involuntarias de la lectura de los arts. 20 y 21 de la Ley 26657, puede advertirse que la internación es realizada por el equipo médico y posteriormente comunicada al juez y al órgano de revisión dentro del plazo de diez horas, quien puede autorizar o denegar la internación o, en su caso, requerir informes ampliatorios, pudiendo ordenar la internación de oficio cuando, dados los requisitos marcados por la ley, el servicio de salud responsable de la cobertura se negase a realizarla. 


\section{c. Visión interdisciplinaria}

Es precisa la ley nacional cuando establece que debe promoverse que la atención en salud mental esté a cargo de un equipo interdisciplinario integrado por profesionales, técnicos y otros trabajadores capacitados con la debida acreditación de autoridad competente, tales como los pertenecientes a las áreas de psicología, psiquiatría, trabajo social, enfermería, terapia ocupacional y otras disciplinas.

En esta línea, la ley nos habla de las nuevas modalidades de abordaje que deben incluirse en forma paulatina en el tratamiento del paciente mental tales como: operadores comunitarios, acompañantes terapéuticos, psicoterapeutas de familias y de grupos, rehabilitadores y facilitadores de actividades socio-laborales, culturales, artísticas y recreativas; estableciendo la reglamentación que los integrantes de dichos equipos responden en función de sus propias incumbencias en el marco del trabajo conjunto.

Para ordenar la internación de un paciente, se requiere la evaluación o diagnóstico interdisciplinario e integral con la firma de, por los menos, dos profesionales, los que necesariamente deberán ser psicólogo o médico psiquiatra (art. 20 de la ley nacional 26.657).

\section{d. Derechos de los pacientes}

Siguiendo con el análisis de la nueva normativa, debemos remarcar que propone el resguardo de los derechos del paciente mental (art.11 de la ley nacional); con la debida participación de los familiares del mismo y otros actores sociales como las ONG etc. (art. 10), entendidos todos como usuarios del sistema de salud mental y haciendo especial hincapié en los derechos de pacientes niños y adolescentes (art.12).

Estos principios responden a la Ley 26.529 (19.11.09) sobre derechos del paciente en su relación con los profesionales e instituciones de la salud, como así también a la Declaración Universal sobre Bioética y Derechos Humanos, Brasilia, 2005; resultando también de aplicación las reglas de los derechos del consumidor cuando se trate de la atención de la salud mental en la faz privada, ya que el contrato médico se encuentra inmerso en dicha normativa. 


\section{Impactos de la nueva normativa}

Esta nueva normativa, como la mayoría de las leyes que se dictan en nuestro país, suelen no venir acompañada de las decisiones políticas presupuestarias, de infraestructura etc. - que hagan posible que su puesta en funcionamiento logre el éxito de las premisas sustentadas por la misma.

Los resultados obtenidos en el ámbito de la salud han sido buenos, pero no suficientes, ya que - si bien se han simplificado y/o suprimido muchos de los trámites burocráticos que se exigían a los fines de internación y externación de pacientes judicializados - no se han logrado mejoras en el sistema de derivación de pacientes por falta de camas o por ser inadecuado el establecimiento sugerido para la internación. Tampoco se han implementado ni la atención en los hospitales generales de estos tipos de patologías, ni se han creado los organismos intermedios creados por la ley que garantizan un mejor y adecuado tratamiento ambulatorio del mismo.

En realidad, no se trata propiamente de errores estructurales 0 endémicos de la ley ni derivaciones negativas del sistema, sino de fallas que todavía no tienen solución por falta de una política de salud adecuada.

En esta línea de pensamiento podemos mencionar la falta de recursos humanos y económicos lo que, en determinadas épocas, ocasiona una demora considerable en la realización de los diagnósticos interdisciplinarios; la falta de medios o medicamentos para realizar los tratamiento necesarios, la rehabilitación insuficiente, la falta de recursos tecnológicos que permitan una comunicación fluida con el juez etc.

Esto ha logrado que, en muchas provincias, el sistema de salud mental se encuentre colapsado con un aumento constante de pacientes, lo que se ve agravado por las derivaciones que trae aparejada el consumo de droga y alcohol que, a su vez, impactan en temas de violencias domésticas; como así también que, al bajarse la mayoría de edad en nuestro país, se ha aumentado el grupo etario que ingresa al sistema de las internaciones involuntarias.

En el ámbito del Poder Judicial, la nueva normativa devuelve al juez su rol natural, en tanto - conforme la materia de que se trata - actuará primero la Salud y luego la Justicia, acotándose la intervención judicial sólo para los casos de internaciones involuntarias y cuando se existan las condiciones para su 
judicialización, previo a un diagnóstico interdisciplinario y siempre con carácter restrictivo.

Es más, el juez está designado, más que para internar, para controlar que no tenga dicha internación un lapso prolongado innecesario; e, inclusive, el órgano de revisión puede solicitar la intervención judicial así como de la defensa pública ante situaciones irregulares que vayan en desmedro de los derechos de las personas usuarias del servicio de salud mental.

Asimismo, la responsabilidad de la externación vuelve a retomar su rumbo, ya que la decisión de la misma es de neto corte médico-sanatorial y sólo debe ser comunicada al juez que la hubiera ordenado; salvo en los casos del fuero penal donde indefectiblemente sigue rigiendo el art. 34 del Código Penal y el consecuente diagnóstico de dos médicos forenses o legistas.

Sin embargo, debemos señalar la carencia de recursos legales que comprometan a los familiares con el seguimiento y contención del tratamiento ambulatorio (no prevista como obligatoria en la nueva ley a diferencia de la ley 22.914); existiendo muchos casos de abandono del paciente en los institutos, sobre todo cuando se trata de establecimientos públicos.

Podemos agregar que el sistema muchas veces se ve complicado por la falta de uniformidad de criterios de la justicia, como la falta de intervención de la fuerza pública en los casos que resulta necesario, como sería el ingreso por la fuerza pública a los fines de la atención de un paciente en riesgo cierto e inminente.

Por último queremos recalcar como un factor desfavorable, el desconocimiento de la magistratura sobre esta materia y muchas veces la falta de atención urgente de este tema teniendo en cuenta la situación del justiciable y particularmente la vulnerabilidad que se encuentra en juego.

\section{La desjudicialización y la responsabilidad judicial}

Es contundente la doctrina judicial al sostener que, para sustraer a una persona su derecho a la libertad basándose en sus supuestas alteraciones mentales, deben respetarse a ultranza los principios de legalidad (causas tipificadas legalmente) y de no arbitrariedad. Es decir, la afectación de la 
libertad debe resultar compatible con las ideas de razonabilidad, previsibilidad y proporcionalidad.

Desde esta perspectiva, proponemos que se debe analizar, en cada caso en particular, si la decisión judicial que dispone o ratifica una internación forzada tiene sustento en la ley vigente (exigencia de legalidad), y si, en tal circunstancia, se cumple con los principios antes aludidos y los nuevos paradigmas sustentados por la nueva ley en salud mental y su decreto reglamentario.

Muchas decisiones judiciales que disponen internaciones forzosas - o que las ratifican - son incompatibles el ordenamiento jurídico internacional y nacional. En algunos casos, tienen como fundamento legal dictámenes médicos que, con frecuencia, son ambiguos, estandarizados y meramente clasificatorios, concretados luego de fugaces entrevistas con el afectado y que no respetan el diagnóstico interdisciplinario. Dice el decreto reglamentario al respecto que "el informe debería contener conclusiones conjuntas producto de un trabajo interdisciplinario" (Decreto 603/2013).

Debemos remarcar que una de las obligaciones trascendentales del magistrado interviniente es la de promover la externación oportuna, ya se trate de una internación voluntaria o de urgencia, razón por la cual debe adoptar, consecuentemente, todas las medidas a su alcance para que el período de reclusión institucional se limite al tiempo indispensable requerido por las necesidades terapéuticas y la seguridad del internado y de terceros.. Lo contrario implicaría una desprolijidad, que no se condice con la exigencia de evitar internaciones prolongadas sin sustento en la gravedad de la patología que pudiera presentar quien debe ser realmente recluido en una unidad asistencial.

Es por ello que, en los casos en que no se precisen los criterios para ordenar la internación ni se especifiquen los estándares sustantivos mínimos para la reclusión preventiva (definir con precisión el grado de peligrosidad del paciente), debe resultar a todas luces la improcedencia de la medida, que siempre debe tener interpretación restrictiva y, como consecuencia de ello, debe surgir la responsabilidad del órgano que la ordena, ya que no se ha acreditado que la internación del paciente fuera la única solución viable que tenía el Estado para lograr un mejoramiento en la salud mental de aquel. 
Es por ello que podemos aseverar que, en estas situaciones, deviene imperioso contar con un control judicial adecuado acerca de la necesidad de la medida de internación y las condiciones de ésta; obligación que debe cumplirse en intervalos periódicos para garantizar la legalidad de la medida de seguridad privativa de libertad impuesta y evitar que las personas institucionalizadas psiquiátricamente no sean escuchadas "y vistas" por el sistema judicial.

De lo contrario aparecerá severamente comprometida la responsabilidad del Estado.

\section{Conclusiones}

En primer lugar - si bien la ley en salud mental es exquisita en sus términos y valedera como sistema de protección al paciente - se desvanece ante la falta de acompañamiento de una política adecuada del Estado. Y hablamos del Estado en general, porque es un tema que no compete solo a salud sino también a todos los organismos estatales que deben acompañar en este proceso de humanización de la salud mental.

Es por ello que, amén de la quimera de contar con mayor presupuesto a nivel de salud pública, todos los poderes públicos deben tomar conciencia de articular los programas de prevención y promoción de la ley, sobre todo en materia de consumo de drogas y alcohol o en los centros de prevención de violencia doméstica y cada funcionario o sector desde el lugar que ocupa.

En segundo término, se deben discutir y plasmar verdaderas políticas públicas en salud mental, conscientes que la misma es un deber que el Estado debe garantizar.

En tercer lugar, trabajar a través de oficinas de derechos humanos con psicólogos, trabajadores sociales etc., desde la premisa de la vulnerabilidad del paciente con padecimiento mental, sobre la importancia de la contención familiar y las posibilidades de externación, unidos a la creación de los lugares de cuidados intermedios referenciados y mecanismos de reinserción social (como programas laborales especiales, planes de vivienda etc.).

Por otro lado, si bien debe garantizarse la desjudicialización de las internaciones psiquiátricas - con la idea de que la misma sea aplicable cuando, existiendo un diagnóstico de riesgo cierto e inminente, resulte ser la medida 
terapéutica necesaria e inevitable - desde que ello no signifique consagrar la irresponsabilidad del sistema judicial.

Es por ello que debemos bregar para que el Poder Judicial y el Estado se erijan como los guardianes de los derechos humanos en el marco de las internaciones involuntarias judicializadas, compartiendo la doctrina que señala que: "... los abogados y magistrados debemos gestar criterios jurídicos y pautas que sostengan fallos meditados y fundados con los recaudos procesales imprescindibles [...] habida cuenta que, los causantes en los procesos de internación psiquiátrica merecen, al menos, la misma protección que poseen los involucrados en cualquier tipo de proceso" (Kraut, 2008, p. 7) y reconociendo que, si bien no debe propugnarse la judicialización total de las personas con padecimientos mentales, tampoco resulta factible eliminar el control judicial cuando se restringe la libertad y se puede afectar la integridad física y mental del individuo (Diana, 2007).

La desjudicialización no debe desprenderse de la doctrina judicial sustentada por el Máximo Tribunal Nacional que establece que "el derecho debe ejercer una función preventiva y tuitiva de los derechos fundamentales de la persona con sufrimiento mental, cumpliendo para ello un rol preponderante la actividad jurisdiccional" (CSNJ, 2008 "R.M.J")

En definitiva, debemos señalar que el rol principal del Poder Judicial consiste en ser custodio de los derechos fundamentales de los ciudadanos con patologías psiquiátricas, por lo que, lejos de tratarse de una función de ejecución, la misma debe ser garantista y de control o fiscalización; con la consiguiente responsabilidad que puede traer aparejada al Estado el no asumir la importancia de la protección del enfermo mental a la luz de los derechos humanos.

\section{Referencias}

ARGENTINA, Decreto 603, de 28 de mayo 2013. Reglamenta Ley 26657. Boletín Oficial de 29 de mayo 2013. [on line] Disponible en http://www.infoleg.gob.ar/infolegInternet/verNorma.do?id=215485 (fecha de consulta 8 set. 2014)

ARGENTINA. Ley 26.657, de 25 de noviembre 2010. Derecho a la Protección de la Salud Mental. Disposiciones complementarias. Derógase la Ley № 22.91 Boletín Oficial de 3 de diciembre de 2010. [on line] Disponible en 
http://www.infoleg.gob.ar/infoleglnternet/verNorma.do;jsessionid=F0562C4EAD F340730038293F7D9BC838?id=175977 (fecha de consulta 8 set. 2014)

ARGENTINA, Ley 22.914, de 15 de setiembre 1983.Regúlase la internación de personas en establecimientos de salud mental, públicos o privados, y de alcohólicos crónicos y toxicómanos en establecimientos adecuados, también públicos o privados. Ambitos de aplicación. Boletín Oficial de 20 de setiembre de 1983. [on line] Disponible en: http://www.infoleg.gob.ar/infolegInternet/verNorma.do?id=175978 (fecha de consulta 8 set. 2014)

ARGENTINA, Ley 26.529, de 21 de octubre 2009. Derechos del Paciente en su Relación con los Profesionales e Instituciones de la Salud. Boletín Oficial de 20 de noviembre de 2009. [on line] Disponible en: http://www.infoleg.gov.ar/infolegInternet/anexos/160000164999/160432/norma.htm. (fecha de consulta 8 set. 2014)

AVALOS, Antonio. La internación psiquiátrica involuntaria en la Provincia de Córdoba. Tesis (Magister) Córdoba, Universidad Nacional de La Plata, Facultad de Medicina, 2008. [on line] Disponible en: http://www.postgradofcm.edu.ar/ProduccionCientifica/TesisMagisters/5.pdf (fecha de consulta 8 set. 2014)

CARDENAS, Eduardo y GRIMSON Ricardoy. En: ÁLVAREZ, José A. El juicio de insana y la internación psiquiátrica. Buenos Aires: Astrea, 1985. p. 164 ISBN 9505081561

DIANA, Nicolás. El respeto de las personas con padecimientos mentales. En la soledad del olvido. Lexis, № 0003/013585 [on line] Disponible en: https://www.google.com.br/url? sa=t\&rct=i\&q=\&esrc=s\&source=web\&cd=2\&ved =0CCYQFjAB\&url=https\%3A\%2F\%2Fwww.jusbaires.gob.ar\%2Fsites\%2Fdefaul t\%2Ffiles\%2Farticulo de nicolas diana.doc\&ei=gKANVOGFJsiyogTH2oLgDQ \&usg=AFQjCNFPBmHSqK7iZ5

JJ2pNX6 SXG6ckA\&sig2=QGD2tFbC20ETqDHBzuxNhg (fecha de consulta 8 set. 2014).

KRAUT, Alfredo Jorge. Algunas reflexiones y propuestas sobre la internación forzosa de pacientes psiquiátricos no delincuentes. Jurisprudencia Argentina, 3(5581):730-740, 17 ago. 1988. [on line] Disponible en: http://www.google.com.br/url?sa=t\&rct=i\&q=\&esrc=s\&source=web\&cd=2\&cad=r ja\&uact=8\&ved =0CCYQFjAB\&url=http\%3A\%2F\%2Fpostgradofadecs. uncoma.e du.ar\%2Farchivos\%2Fsaludmentalflia\%2FPacientes\%2520mentales\%2520Sui cidio\%2520de\%2520un\%2520internado\%2520Sistema\%2520de\%2520puertas abiertas\%2520Kraut\%2520-

\%2520Sosa.docx\&ei=qKINVNOLHJa4ogSn3ICICQ\&usg=AFQjCNFZfSgipW8b HUD74xYyCaO-8VHKIw\&sig2=1 wyrvVcsWWK-nRw5bDblsg (fecha de consulta 8 set. 2014)

OGANIZACIÓN DE NACIONES UNIDAS (ONU). Asamblea General de las Naciones Unidas en su Resolución № 46/119 del 17 de diciembre 1991. [on line] Disponible en: https://www.cidh.oas.org/PRIVADAS/principiosproteccionmental.htm (fecha de consulta 8 set. 2014) 
RIVERA, Julio C. y HOOFT, Irene. La nueva ley 26657 de salud mental. Lexis № 0003/015423. [on line] Disponible en:

http://derechosdefamiliaysucesiones.blogspot.com.br/2012/10/salud-mentalrivera-hooft.html (fecha de consulta 8 set. 2014)

TERZAGA, Patricia. Declaración judicial de incapacidad por insana Inhabilitación judicial e internación por medios coactivos. LNC 2008-2-123Lexis № 0003/70042561-1 [on line] Disponible en: www.abeledoperrot.com (fecha de consulta 8 set. 2014).

\section{Referencias Jurisprudenciales:}

ARGENTINA.Corte Suprema de Justicia de la Nación. 19 de febrero de 2008, p 123-147. "R.M.J". Actualidad Jurídica [on line] Disponible en: www.actualidadjuridica.com.ar (fecha de consulta 8 set. 2014). 